\title{
A new software tool for fast and repeatable joint biomechanic analysis
}

\author{
S. Martelli, N. Lopomo \& E. Ferretti \\ Rizzoli Institutes, Lab. Biomeccanica, Bologna, Italy
}

\begin{abstract}
This paper describes a method for a computerized analysis of diarthroid joints. The software implements reliable functions to study the shapes of the articulating surfaces through 2D arbitrary sections and geometrical fitting of lines/conics to quantify ligament behaviour through the computation of elongation, strain and orientation during the recorded motion. The software also allows for the computation of the most classical kinematic parameters, such as helical axes, instantaneous rotations and displacements, centers of rotation or pivot points of motion. In addition, new functional-anatomical features, such as distance maps, contact areas tracking, and tools to validate functional hypothesis on the joint mechanism are possible in an interactive environment which display the 3D behaviour of the acquired or selected joint structure. An easy interface also allows non-expert users to analyse the joint biomechanics and a test-demo is available freeware at http://www.studyjoint.org. This approach represents a generalization of the analysis of several cadaveric joints, and its preliminary versions were used for kinematic and anatomical investigations about normal and pathological knees or shoulders. It provides a standard method for a complete analysis of joints or model predictions.
\end{abstract}

Keywords: joint, biomechanics, kinematics, anatomy, software, model.

\section{Introduction}

The recent development of navigation systems for surgery and orthopaedic treatments and an increased interest for biomechanical modelling require more accurate tools for model validation and more precise knowledge of joint function. This study synthesises a long experience of human joint investigations for biomechanics studies and computer-assisted orthopaedic evaluation, showing 
that it is possible to use a general method to analyse all diarthrodial joints (e.g. human knee, hip, shoulder or elbow).

This paper describes new software which can be used to elaborate of data about the joint motion and anatomical structures. It allows one to study the conformity of bones in the articulating areas, the morphology of the bones and the ligaments' insertions, the elongation or the orientation of ligaments during motion, the joint kinematic features. The software is very flexible and can be used also for partial or specific investigations on the role of a particular tissue on the joint or to validate models predictions on real data.

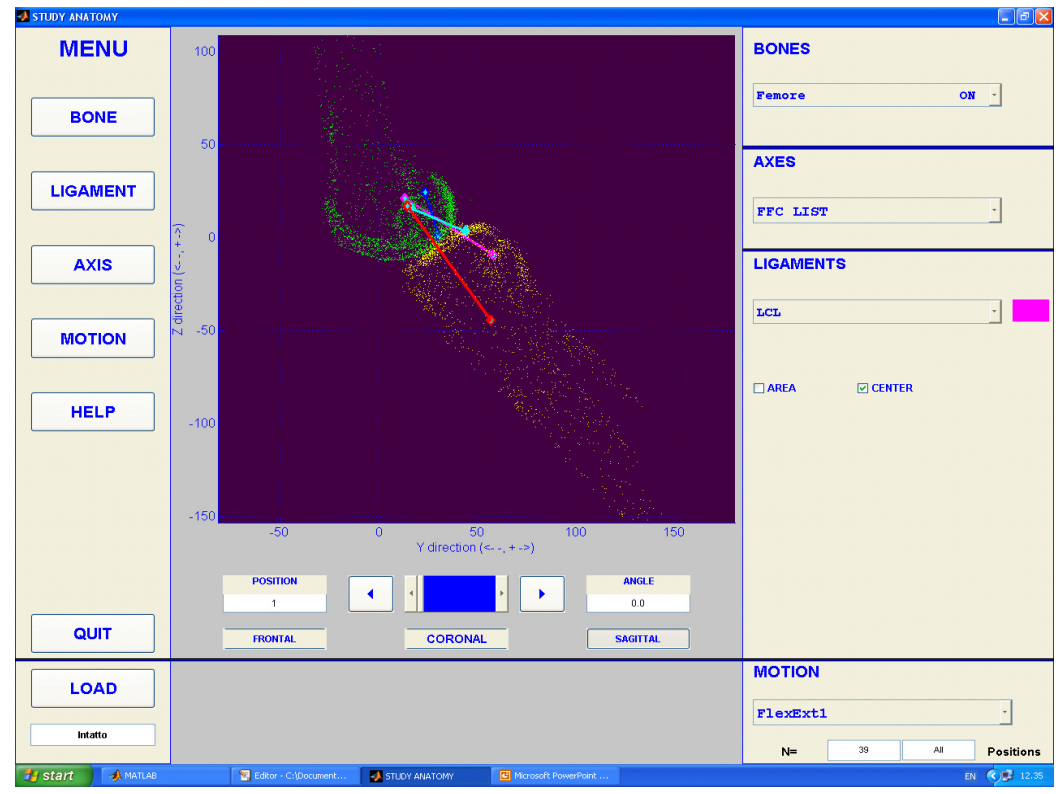

Figure 1: Interface of the software for joint analysis with data of a cadaveric knee acquired by FlashPoint navigation system (Image Guided, Boulder, Colorado, USA). The central windows represent the joint during the passive range of motion and the main ligaments approximated by lines. On the left the menu describing the available data; on the right the command menu grouping the algorithms available for bone, ligament and motion analysis.

\section{Method description}

\subsection{Input data}

The method described in this paper elaborates sets of 3D points and lists of 4X4 matrices representing trajectories in homogeneous coordinates. The clouds of points represent surfaces of rigid bodies fixed to the joint, such as bones, 
boundaries of the insertion areas of ligaments or tendons, and can be acquired by navigation systems or 6 degrees of freedom (dof) electro-goniometers, e.g 5. The list of matrices represent the instantaneous position of the mobile segment of a diarthroid joint with respect to the fixed one, acquired by 6 dof trackers, e.g. 5 .

\subsection{D display}

The software represents the digitized objects during the recorded motion and allows the point-by-point or continuous display of the relative positions of the bones and their subsets, e.g the ligaments insertion areas. Standard zooming and 3D manipulation is available by drag and drop or mouse - buttons. The different objects can be shown or hidden by the display simply clicking of the data menu, as shown on the right in Fig. 1.

\subsection{Tools for bone analysis}

The sets of points classified as "bones" can be analysed in details in 2D sections oriented in the standard clinical views (sagittal, frontal and transversal planes), or in any arbitrary plane obtained rotating or translating them. The $2 \mathrm{D}$ section is displayed in a 2D windows showing profiles of the slices and results particularly suitable for shape analysis, least-square fitting of lines, circles or ellipses, e.g Fig. 2. The profiles can be tracked during motion, to study, for example, contact points or bones sliding.
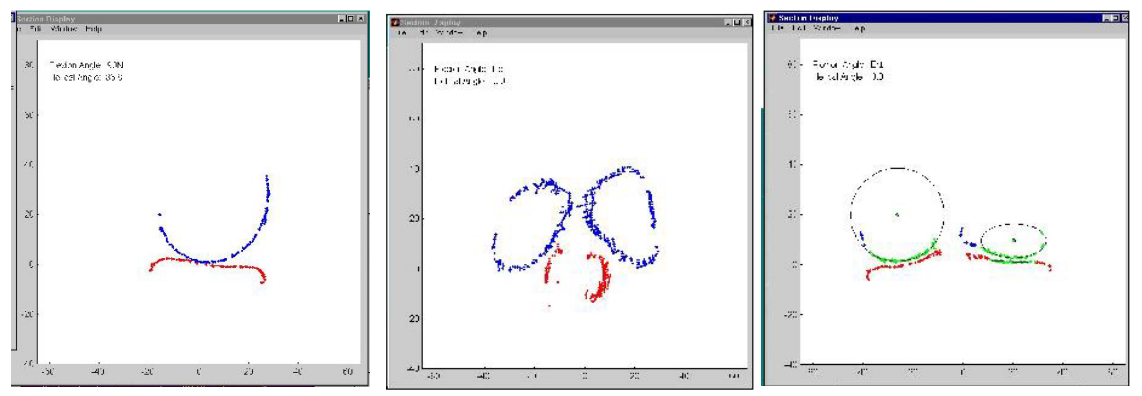

Figure 2: Display of 2D sections of $3 \mathrm{~mm}$ thickness. (a) Sagittal section of the knee medial compartment; (b) transversal section through tibial spine; (c) frontal section of the knee at extension with least square fitting of the femoral condyles (with conics) and the tibial plateau (with a line).

\subsection{Tools for ligaments or tendons analysis}

Ligaments are structures defined by two insertion areas on bones. The software displays the ligament as a line connecting the centers of these insertions areas. If available among input data, also fibers are displayed as lines joining two points in the insertion areas, e.g. the antero-medial and the postero-lateral bundles of 
the anterior cruciate ligament of the knee. Computations on ligaments fibers include plotting of it length (absolute or percentage) during motion and plotting of the orientation with respect to clinical or user-defines planes.

\subsection{Tools for kinematic analysis}

Recorded motion can be analysed computing instantaneous helical axes and angles, instantaneous rotations with the cardan method (in the standard clinical reference frame or around any user-defined orthogonal axes), instantaneous displacements of user-defined points on the articular segments. Computation of angular and displacement laxity is also supported.

\section{Study Joint implementation}

Study_Joint software was designed in order to optimize both the graphical display and the geometrical and functional computations and was developed in MATLAB language (Mathworks, Inc.), because of its simple coding-debugging and the presence of efficient libraries.

Study_Joint software is made of 99 modules, as shown in Fig. 3, including the main one, and is organized into 6 levels (corresponding to columns in Fig. 3). The first level is the interface creation, in particular modules corresponding to the buttons callbacks (at bottom of column 1) or functions drawing the interface panels (bone_menu, lig_menu, axis_menu, ... load_data). Study_Joint interface was entirely developed using MĀTLAB GUIDE (Graphical User Interface Development Environment), but the 3D and 2D rendering of input objects or plot and sections were managed directly, by 3Ddisplay module (second column), displaySection and window2d_proj, plot_elongation, plot_function (second and third column) respectively. These windows have custom context menus associated to the mouse right button (for managing 3D windows handling, window settings, plotting scale, printing and saving of images) and customized refresh functions optimized for the input structures. It has to be noticed that the most efficient management of 3D surface drawing (both for the rendering quality and speed) was obtained using openGL libraries.

The remaining modules are used for parameters management and for computations. Numerical algorithms are often simple and implemented using a simple MATLAB functions (e.g. elongation is implemented computing vectors norm, projections are obtained multiplying projection matrix and $3 \mathrm{D}$ points), and the software flow is linear, with no nested or recursive module, and uses at most 3 level of computations. Basically the most time-consuming computations correspond to least square algorithms, distance minimization or plotting management and loading of numerous structures rather than pure reckoning.

The most time-consuming function of the software resulted findMinDist, which is used to compute distance maps between two sets of points and whose execution time depends both on the input data dimension and the user - defined tolerance (which increases the output data dimension). The execution time of this function is closely connected with input and output growth, because of the dramatic raise of memory use. 


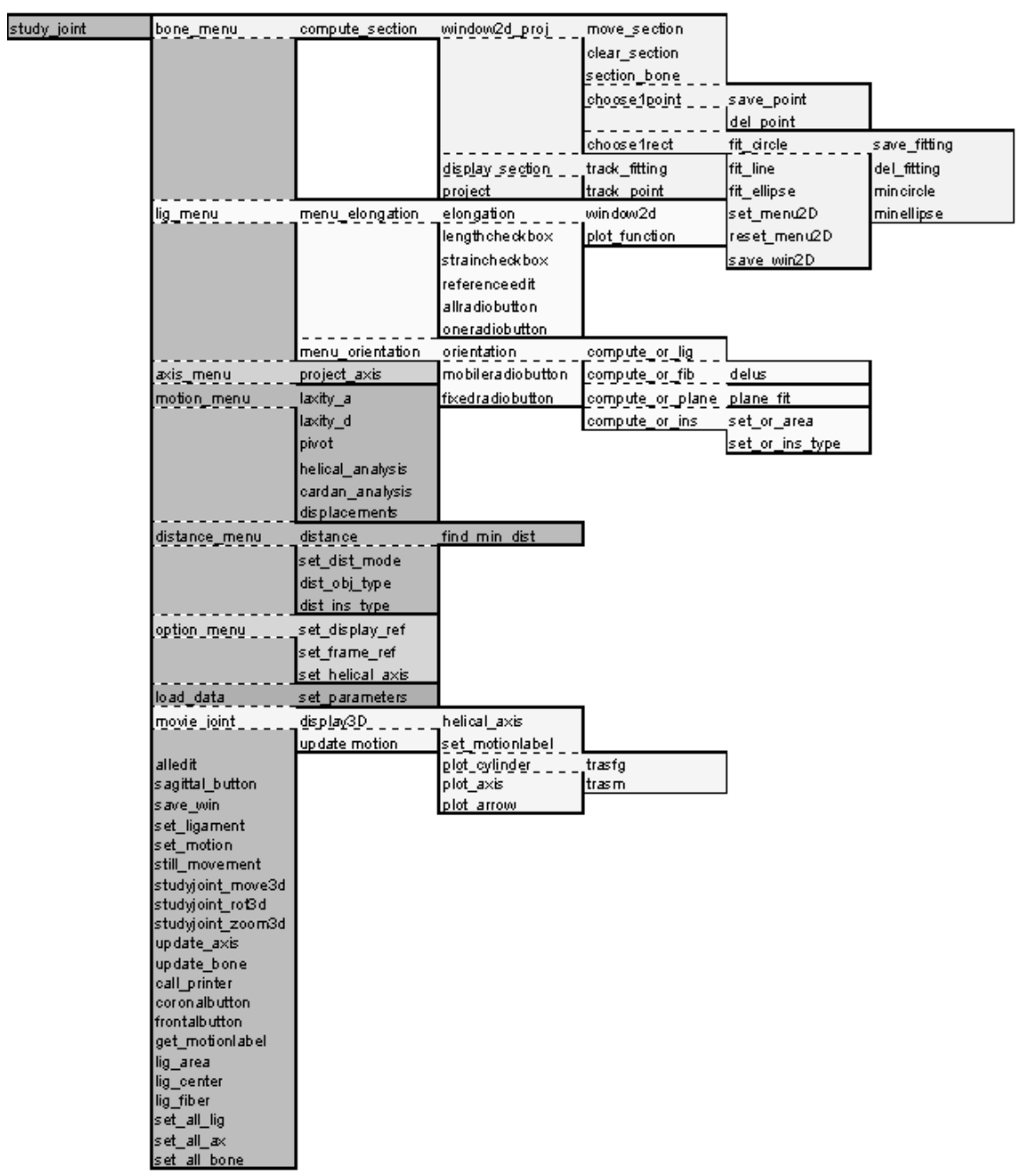

Figure 3: Study_Joint functions architecture.

We also compiled the original MATLAB code (with the standard MATLAB compiler, release 7) in order to obtain a $\mathrm{C}++$ code and an executable file. This application resulted very portable on different computer and screen features, but the execution time was comparable with the MATLAB software in nearly all cases. MATLAB application provided superior performances when the dimension of the input data and computation matrices-vectors increased, while the optimized $\mathrm{C}$ code performed better when a large amount of bit manipulation was required. In almost all cases, both with relatively large amount of input data from cadaver tests and with small amount of data for specific applications, Study_Joint showed similar performances from the MATLAB code or from the 
compiled one, and the execution time was always compatible with real-time elaborations (for almost all the functions the execution's times are under 0.3 seconds).

At present both the MATLAB and compiled version of Study_Joint are used with input data recorded as MATLAB structures, because this format allows a fast and optimal management of loading, saving and storing operations.

\section{Experimental results}

The different parts of the software have been used for previous studies on knee and shoulder joints, $1-4$, and successfully compared with magnetic resonance and roetenger stereo-photogrammetric analysis in 6 .

\section{Discussion and conclusions}

The method allows an exhaustive analysis of joints, from anatomical and kinematic point of view. This method suggests a standard way to investigate joints or to validate a model prediction, starting from the morphological constraints due to bone shapes and then considering the main kinematic features to identify the best physical model of the joint. Ligaments are carefully examined, as we believe their role as secondary constraint or stabilizer can be deduced from the observation of their behaviour during motion.

This method has resulted very effective and user-friendly in medical applications and in-vivo investigations. We plan to use it for in-vivo analysis during computer-assisted surgery.

\section{References}

[1] Martelli S., Acquaroli F., Pinskerova V., Spettoli A., Visani A., An Anatomical Model of the Knee Joint Obtained by Computer Dissection, in Lecture Notes in Computer Science n.2489 (LNCS 2489), Proceedings of V MICCAI 2002 , eds. T. Dohi and R. Kikinis (Springer, 2002) pp. 308-314

[2] Martelli S., Visani A., Computer Investigation into the anatomical Location of the Axes of Rotation in the Knee, J. Mechanics in Medicine \& Biology, 2, n. 3\&4 (2002) 433-447

[3] Zaffagnini S., Martelli S., Acquaroli F., Computer investigation of ACL orientation during passive range of motion, Computers in Biology and Medicine, 34 , n.2 (2004) $153-163$

[4] Martelli S., Zaffagnini S., Minola R., Golano P., Giovannucci D., Computerized kinematic analysis of rotator cuff behaviour during passive motion, in Proc. of CAOS 2004, Chicago (June 2004), pp.349-350

[5] Martelli S., Zaffagnini S., Computerized Protocol for Anatomical and Functional Studies of Joints, in Cartilage and Osteoarthritis, Vol. 2 Structure and In vivo Analysis, SERIE "Methods in Molecular Medicine", eds. F. De 
Ceuninck, M. Sabatini, P. Pastoureau (Humana Press, Totowa, New Jersey, USA, 2004) chap. 17, pp. 323-342

[6] S. Martelli, V. Pinskerova, The shapes of the tibial and femoral articular surfaces in relation to tibiofemoral movement, J Bone and Joint Surg $[\mathrm{Br}]$, 84-B,n.4, (2002) 607- 613 